\title{
ANISOTROPIC PROPERTIES OF THE UPPER MANTLE IN CENTRAL ASIA ACCORDING TO THE GROUP VELOCITY DISPERSION CURVES FOR RAYLEIGH AND LOVE WAVES
}

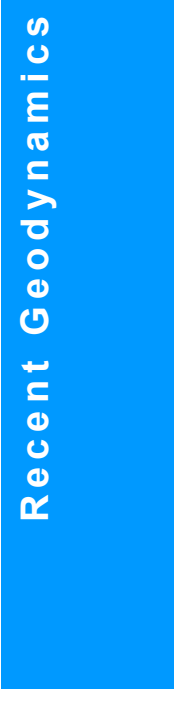

\author{
A. I. Seredkina, O. A. Solovey \\ Institute of the Earth's Crust, Siberian Branch of RAS, Irkutsk, Russia
}

\begin{abstract}
The article presents the results of the study focused on the anisotropic properties of the upper mantle in Central Asia. The study is based on a representative set of the group velocity dispersion curves for Rayleigh and Love waves. The dispersion curves were calculated in the range of 10-250 s. The maps of group velocity distribution patterns and the horizontal resolution estimates were calculated by the surface-wave tomography method developed for a spherical surface. Based on the maps, the local group velocity dispersion curves were reconstructed for the given points within the study region, which were then converted into the one-dimensional velocity sections of $S V$ - and $S H$-waves, and a vertical anisotropy coefficient was estimated. A three-dimensional anisotropic model shows the velocity distribution pattern of $S$-waves in the crust and the mantle to the depth of $500 \mathrm{~km}$. According to this model, vertical anisotropy in the upper mantle is observed to the depth of about $250 \mathrm{~km}$ and has maximum values in the depth interval from the crustal bottom to $150 \mathrm{~km}$. The anisotropic properties are unevenly distributed and reflect the geological structure of the study area. Therefore, tectonically active regions are characterized by the high values of the anisotropy coefficient and the reduced values of the $S$-wave velocities. The presented results can contribute to the further development of more detailed and strictly proved geodynamic models of the study area.
\end{abstract}

Key words: Rayleigh and Love waves; group velocities; surface-wave tomography; vertical anisotropy; upper mantle; Central Asia

\section{RESEARCH ARTICLE}

Handling Editor: K.Zh. Seminsky
Received: October 25, 2017

Revised: January 22, 2018

Accepted: April 3, 2018

For citation: Seredkina A.I., Solovey O.A., 2018. Anisotropic properties of the upper mantle in Central Asia according to the group velocity dispersion curves for Rayleigh and Love waves. Geodynamics \& Tectonophysics 9 (2), 427-437. doi:10.5800/GT-2018-9-2-0354.

Для цитирования: Середкина А.И., Соловей О.А. Анизотропные свойства верхней мантии Центральной Азии по данным дисперсии групповых скоростей волн Рэлея и Лява // Геодинамика и тектонофизика. 2018. Т. 9. № 2. C. 427-437. doi:10.5800/GT-2018-9-20354. 


\title{
АНИЗОТРОПНЫЕ СВОЙСТВА ВЕРХНЕЙ МАНТИИ ЦЕНТРАЛЬНОЙ АЗИИ ПО ДАННЫМ ДИСПЕРСИИ ГРУППОВЫХ СКОРОСТЕЙ ВОЛН РэЛЕЯ И ЛЯВА
}

\author{
А. И. Середкина, о. А. Соловей
}

Институт земной коры СО РАН, Иркутск, Россия

\begin{abstract}
Аннотация: В работе представлены результаты исследования анизотропных свойств верхней мантии Центральной Азии, выполненного на основании представительной выборки дисперсионных кривых групповых скоростей основной моды волн Рэлея и Лява. Дисперсионные кривые рассчитывались в диапазоне периодов 10-250 с. Карты распределений групповых скоростей с оценками горизонтального разрешения вычислялись методом поверхностно-волновой томографии для сферической поверхности. По результатам картирования в заданных с учетом разрешения точках области исследования восстанавливались локальные дисперсионные кривые групповых скоростей и проводилась их инверсия в одномерные скоростные разрезы волн $S V$ и $S H$ и оценивался коэффициент вертикальной анизотропии. Таким образом, была получена трехмерная анизотропная модель распределения скоростей волн $S$ в коре и мантии до глубины 500 км. Показано, что вертикальная анизотропия в верхней мантии наблюдается до глубины около 250 км, с максимумом в интервале глубин от подошвы коры до 150 км. Распределение анизотропных свойств является неоднородным и отражает геологическое строение исследуемой области. Так, тектонически активные регионы характеризуются высокими значениями коэффициента анизотропии и пониженными значениями скоростей $S$-волн. Полученные результаты в дальнейшем могут способствовать построению более детальных и обоснованных геодинамических моделей рассматриваемой территории.
\end{abstract}

Ключевые слова: волны Рэлея и Лява; групповые скорости; поверхностно-волновая томография; вертикальная анизотропия; верхняя мантия; Центральная Азия

\section{1. ВВЕДЕНИЕ}

В настоящее время установлено, что верхняя мантия Земли обладает анизотропными свойствами. По данным поверхностных волн обнаруживается как азимутальная [Forsyth, 1975; Trampert, Woodhouse, 2003], так и вертикальная анизотропия скоростей сейсмических волн [Yanovskaya, Kozhevnikov, 2006; Chen et al., 2009; Dziewonski, Anderson, 1981; Shapiro, Ritzwoller, 2002; Villaseñor et al., 2001; Zhou et $a l ., 2006]$. Вертикальная анизотропия, проявляющаяся в различии скоростей волн $S V$ и $S H$, поляризованных в вертикальной и горизонтальной плоскостях и получаемых путем инверсии дисперсионных кривых волн Рэлея и Лява, соответственно, является неотъемлемым свойством мантии Земли. Так, в стандартной модели PREM значение коэффициента вертикальной анизотропии в подкоровом слое мантии принимается равным $4.8 \%$, а глубже 220 км вещество мантии считается изотропным [Dziewonski, Anderson, 1981].

Несмотря на то, что глубинное строение верхней мантии Центральной Азии достаточно хорошо изучено различными методами [Bijwaard et al., 1998; Koulakov, Bushenkova, 2010; Li et al., 2013; Pandey et al.,
2014; Yanovskaya, Kozhevnikov, 2003; Zhao et al., 2006; u $\partial p$.], количество работ, в которых учитывается вертикальная анизотропия верхней мантии, невелико, причем большинство из них - глобальные модели [Shapiro, Ritzwoller, 2002; Zhou et al., 2006], характеризующиеся невысоким горизонтальным разрешением. Кроме того, результаты немногочисленных региональных [Yanovskaya, Kozhevnikov, 2006; Yanovskaya, Akchurin, 2009; Chen et al., 2009] и глобальных исследований существенно различаются между собой, в том числе и по такому принципиальному вопросу, как существование отрицательной вертикальной анизотропии $\left(\mathrm{V}_{\mathrm{SV}}>\mathrm{V}_{\mathrm{SH}}\right)$.

В данной работе представлены результаты исследования вертикальной анизотропии верхней мантии Центральной Азии, полученные в рамках изучения Азиатского континента по поверхностным волнам [Kozhevnikov et al., 2014; Seredkina et al., 2016]. Область исследования ограничена по долготе $80-130^{\circ}$ Е и по широте $40-60^{\circ} \mathrm{N}$ и состоит из различных в тектоническом отношении регионов (рис. 1). Ее северная часть представлена стабильными Западно-Сибирской плитой и Сибирской платформой. Центральная и южная части охватывают Монголо-Охотского складчатый пояс: высоко- 


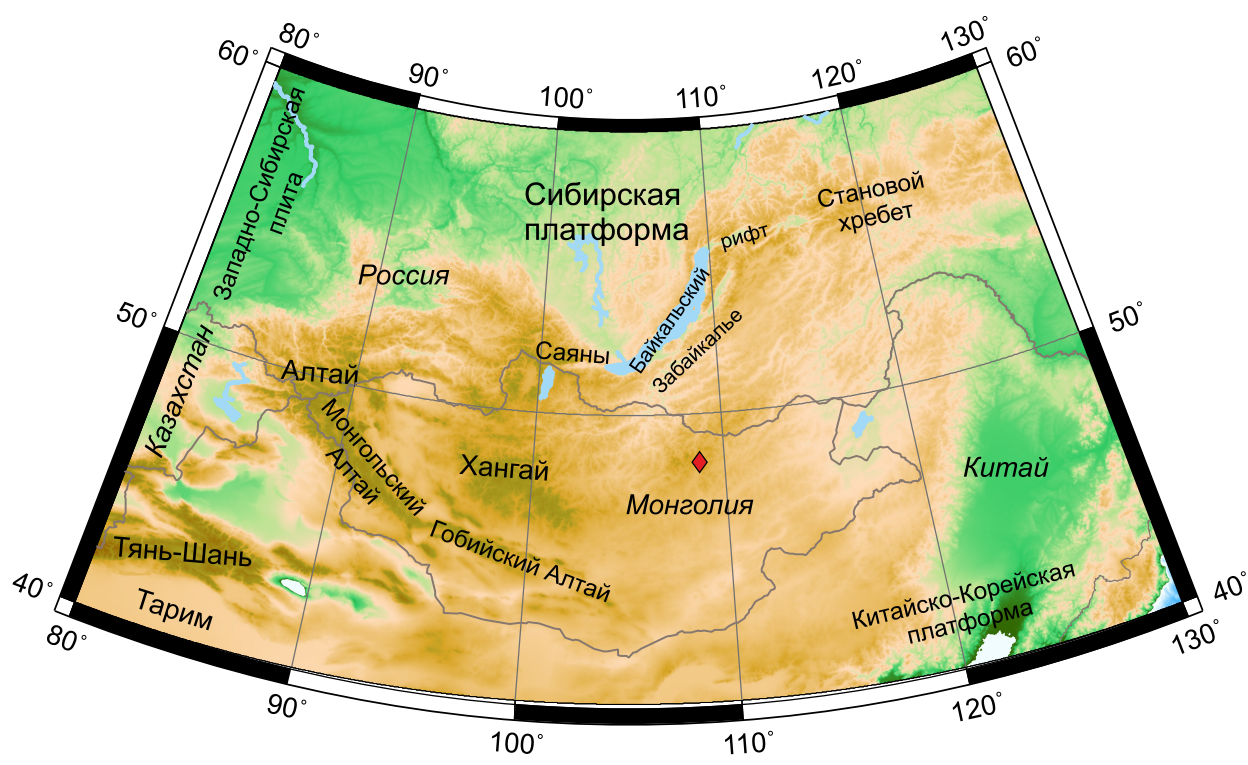

Рис. 1. Область исследования. Рельеф приведен по модели ЕТОРО1 [Amante, Eakins, 2009]. Красным ромбом обозначена точка, для которой показан пример решения одномерной обратной задачи (см. текст).

Fig. 1. Study area. The relief is shown according to the ETOPO1 model [Amante, Eakins, 2009]. A red diamond marks the point for which an example of solving the one-dimensional inverse problem is shown (see the text).

активные в сейсмическом отношении структуры Байкальскую рифтовую зону, высокогорные сооружения Южной Сибири и Западной Монголии, а также складчатые сооружения Забайкалья, Центральной и Восточной Монголии, характеризующиеся в настоящее время процессами умеренного горообразования и рассеянной сейсмичностью. На юговостоке в пределы исследуемой области также частично входит Китайско-Корейская платформа.

\section{2. ИСХОДНЫЕ ДАННЫЕ И МЕТОДЫ ИССЛЕДОВАНИЯ}

Исходными данными для исследования служили результаты картирования групповых скоростей волн Рэлея [Kozhevnikov et al., 2014] и трехмерная модель распределения $S V$-волн в коре и верхней мантии (до глубины 500 км) Центральной Азии [Seredkina et al., 2016], полученные авторами ранее, а также записи волн Лява от сильных $(M \geq 5.5)$ удаленных землетрясений на каналах LHT цифровых широкополосных сейсмических станций сетей IRIS (рис. 2, a). Всего были использованы записи 145 землетрясений, зарегистрированных в период с 1991 по 2009 г. (рис. 2, a). Диапазон расстояний от эпицентров выбранных для анализа землетрясений до регистрирующих станций составил 150016000 км, что позволило выделить основную моду волн Лява в пределах диапазона периодов колебаний от 10 до 250 c.

Вычисление групповых скоростей осуществлялось методом спектрально-временного анализа, представляющего собой аналог многоканальной узкополосной фильтрации [Levshin et al., 1986; Ritzwoller, Levshin, 1998]. Для анализа использовались только записи с высоким отношением сигнал/шум. В результате были получены дисперсионные кривые групповых скоростей волн Лява для 3270 сейсмических трасс, практически равномерно пересекающих рассматриваемую область в различных направлениях (рис. 2, б). Оценка ошибок определения значений групповых скоростей осуществлялась по воспроизводимости дисперсионных кривых: дисперсионные кривые для близких трасс усреднялись, и вычислялись среднеквадратичные отклонения (СКО) скоростей на отдельных периодах от их средних значений. Анализ погрешностей определения групповых скоростей показывает, что наименьшие значения СКО ( 0.02$)$ приурочены к интервалу $\mathrm{T}=25-150$ с, а на остальных периодах СКО несколько выше. На малых периодах большой разброс в значениях групповых скоростей может быть обусловлен не только ошибками вычислений, но и высокой степенью неоднородности земной коры, особенно ее верхней части.

Исследование анизотропных свойств осуществлялось в два этапа. На первом этапе было проведено картирование групповых скоростей волн Лява методом двумерной томографии для сферической поверхности [Yanovskaya, 2001, 2015; Yanovskaya et $a l ., 2000]$. Согласно используемой методике, карты вычислялись отдельно для каждого из заданных периодов колебаний. Всего, таким образом, было построено 16 карт с переменным по периоду ша- 

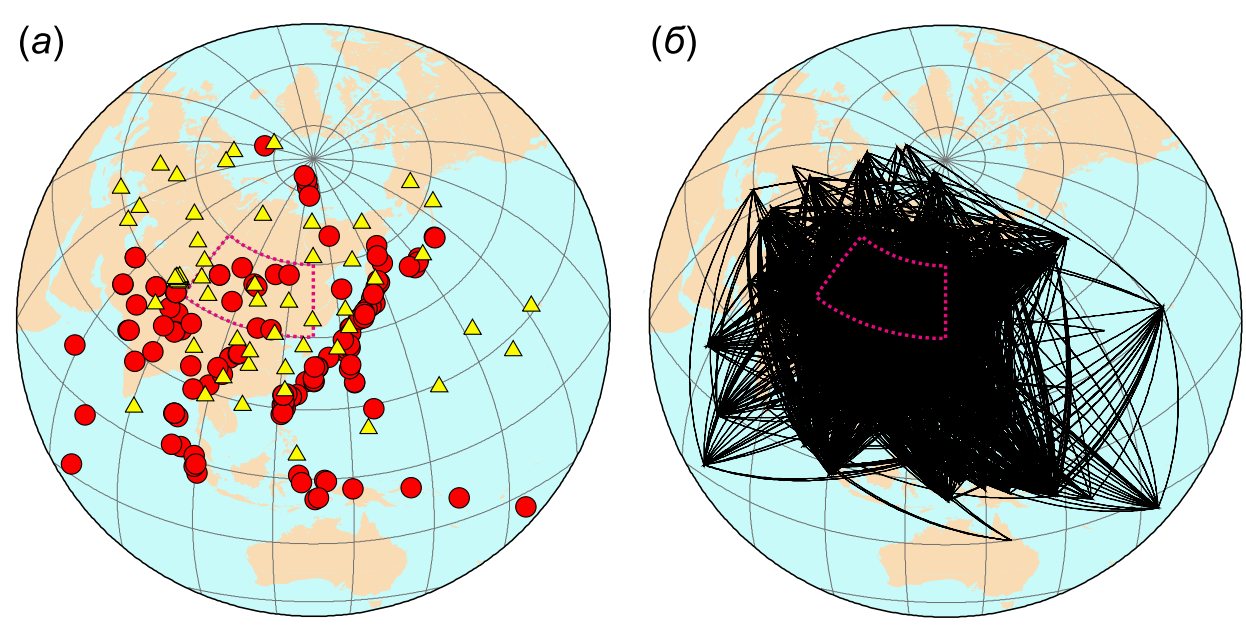

Рис. 2. Используемые для анализа землетрясения (кружки) и сейсмические станции (треугольники) (a); сейсмические трассы для волн Лява (б). Область исследования обозначена розовой штриховкой.

Fig. 2. Analyzed earthquakes (circles), seismic stations (triangles) (a); seismic traces for Love waves (б). The study area is marked by pink hatching.

гом. Для интервала периодов колебаний от 10 до 100 с этот шаг составлял 10 с, а от 100 до 250 с - 25 с, в связи с более монотонным поведением дисперсионных кривых на больших периодах. Для каждой из карт были проведены оценки разрешения результатов картирования путем вычисления эффективного радиуса осреднения $\mathrm{R}$, который зависит главным образом от плотности покрытия того или иного участка области исследования сейсмическими трассами [Yanovskaya, 2001, 2015]. Проведенные ранее синтетические тесты показали [Yanovskaya, 2015], что значения радиуса эквивалентной области сглаживания хорошо согласуются с результатами, полученными методом «шахматной доски». Согласно проведенным нами оценкам, наилучшим разрешением (300-400 км) характеризуются структуры Монголо-Охотского складчатого пояса, в то время как для Сибирской платформы значения эффективного радиуса осреднения несколько выше (рис. 3). Также следует отметить ухудшение разрешения с увеличением периода, связанное с уменьшением количества используемых для анализа сейсмических трасс. Аналогичные результаты ранее были получены авторами при картировании групповых скоростей волн Рэлея [Kozhevnikov et al., 2014], хотя в целом для волн Лява разрешение несколько хуже.

На втором этапе решалась одномерная обратная задача восстановления скоростных разрезов волн SH по дисперсионным кривым волн Лява. Для этого по полученным картам распределений групповых скоростей волн Лява в узлах сетки, выбранных с учетом разрешения, строились локальные дисперсионные кривые. Расчет параметров модели среды, удовлетворяющих дисперсионным кривым, осуществлялся методом сопряженных градиентов
[Yanovskaya, 2015] для модели с двумя плоскопараллельными слоями коры и девятью слоями мантии с линейным изменением скорости с глубиной на полупространстве. В качестве начального приближения использовались скоростные разрезы волн $S V$, полученные авторами ранее [Seredkina et al., 2016]. Для проверки устойчивости полученного решения далее рассчитывался усредненный скоростной разрез $\left(\mathrm{V}_{\mathrm{cp}}=\left(\mathrm{V}_{\mathrm{SH}}+\mathrm{V}_{\mathrm{SV}}\right) / 2\right)$, от которого снова проводились вычисления для обоих типов поверхностных волн. Во всех случаях скоростные разрезы, рассчитанные от обоих начальных приближений, практически совпадали между собой, что свидетельствует о их устойчивости. Пример решения одномерной обратной задачи приведен на рис. 4. Коэффициент анизотропии оценивался как разница скоростей волн $S H$ и $S V$, отнесенная к среднему скоростному разрезу $\left(\alpha=\left(\mathrm{V}_{\mathrm{SH}}-\mathrm{V}_{\mathrm{SV}}\right) / \mathrm{V}_{\mathrm{cp}}\right)$.

\section{3. РЕЗУЛЬТАТЫ И ИХ ОБСУЖДЕНИЕ}

Примеры распределений групповых скоростей волн Лява для периодов колебаний 20, 50, 100 и 150 с показаны на рис. 5. В целом, данные карты демонстрируют те же тенденции, что и распределения групповых скоростей волн Рэлея (рис. 6, a) [Kozhevnikov et al., 2014], а именно высокие скорости поверхностных волн приурочены к платформенным и субплатформенным регионам, тектонически активные структуры (области интенсивного горообразования на юге Сибири и западе Монголии) проявлены как области пониженных групповых скоростей. Однако есть и отличия, которые могут быть обусловлены различной разрешающей 

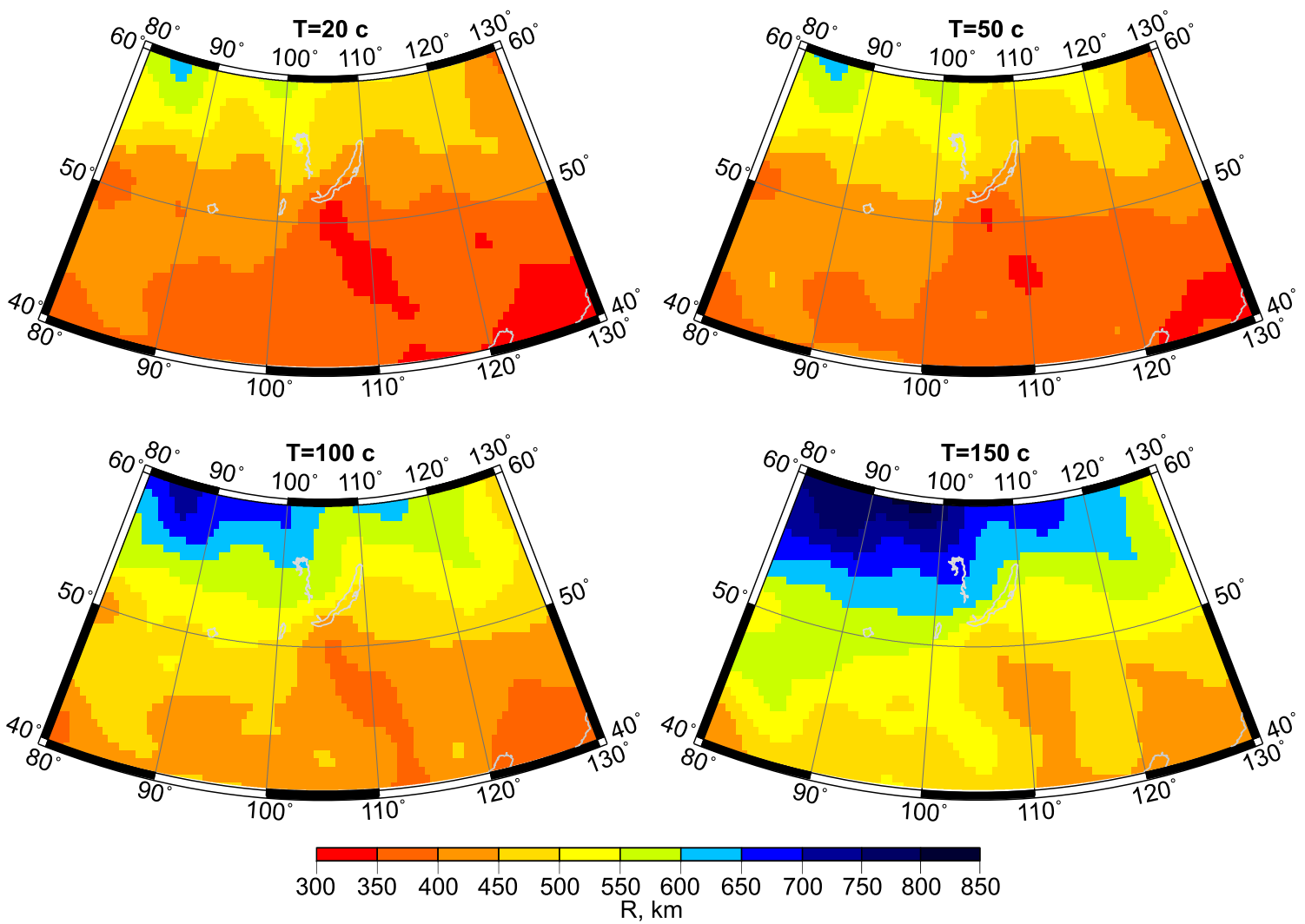

Рис. 3. Карты распределений эффективного радиуса осреднения (R, км) для волн Лява. Над каждой картой указан соответствующий период.

Fig. 3. Distribution maps of the effective averaging radius $(\mathrm{R}, \mathrm{km})$ for Love waves. Corresponding periods are shown above each map.

(a)

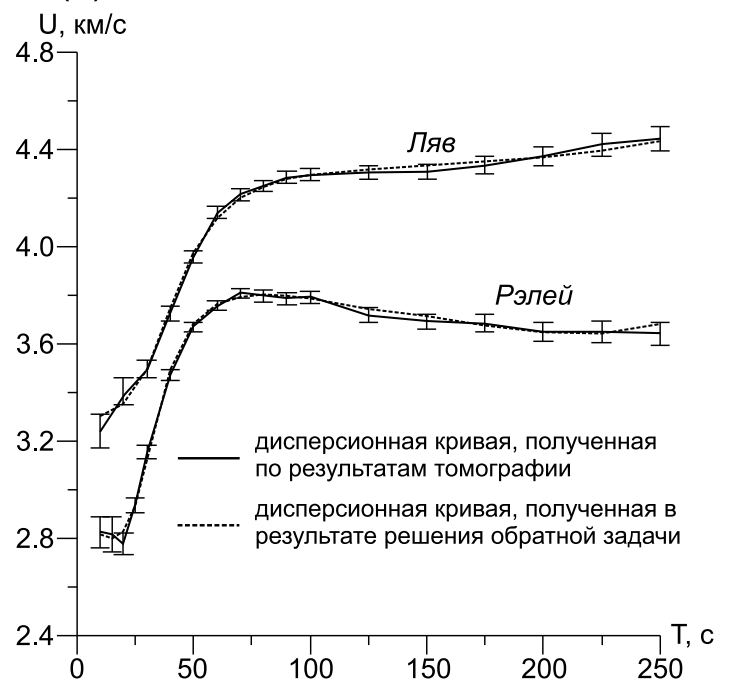

(б)

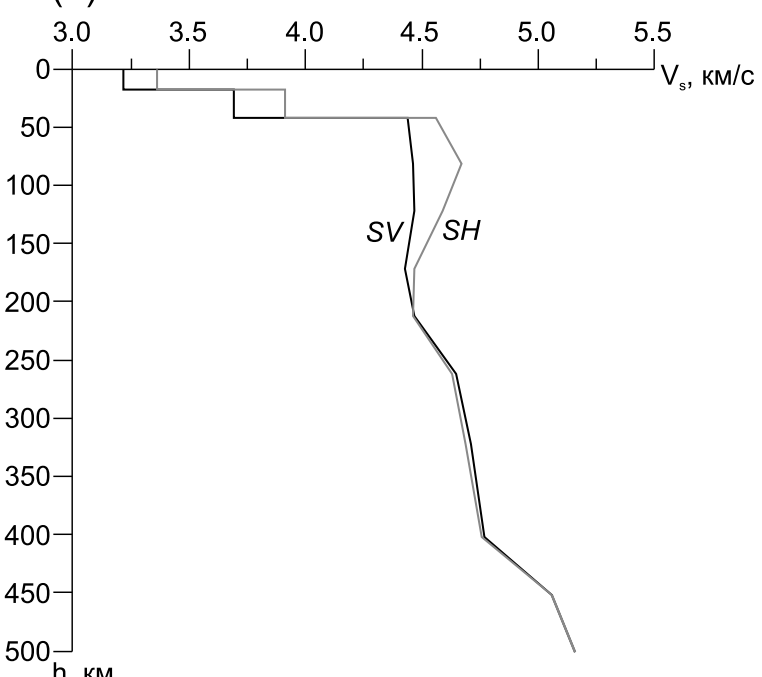

Рис. 4. Пример решения одномерной обратной задачи для точки с координатами $48^{\circ} \mathrm{N}, 109^{\circ} \mathrm{E}$ : дисперсионные кривые волн Рэлея и Лява с оценками погрешностей определения скоростей $(a)$ и соответствующие им скоростные разрезы волн $S V$ и $S H$ (б).

Fig. 4. An example of solving the one-dimensional inverse problem for a point with coordinates $48^{\circ} \mathrm{N}, 109^{\circ} \mathrm{E}$ : the disperseon curves for Rayleigh and Love waves, and velocity estimation errors (a); and the corresponding $\mathrm{SV}$ - and $\mathrm{SH}$-wave velocity sections (б). 

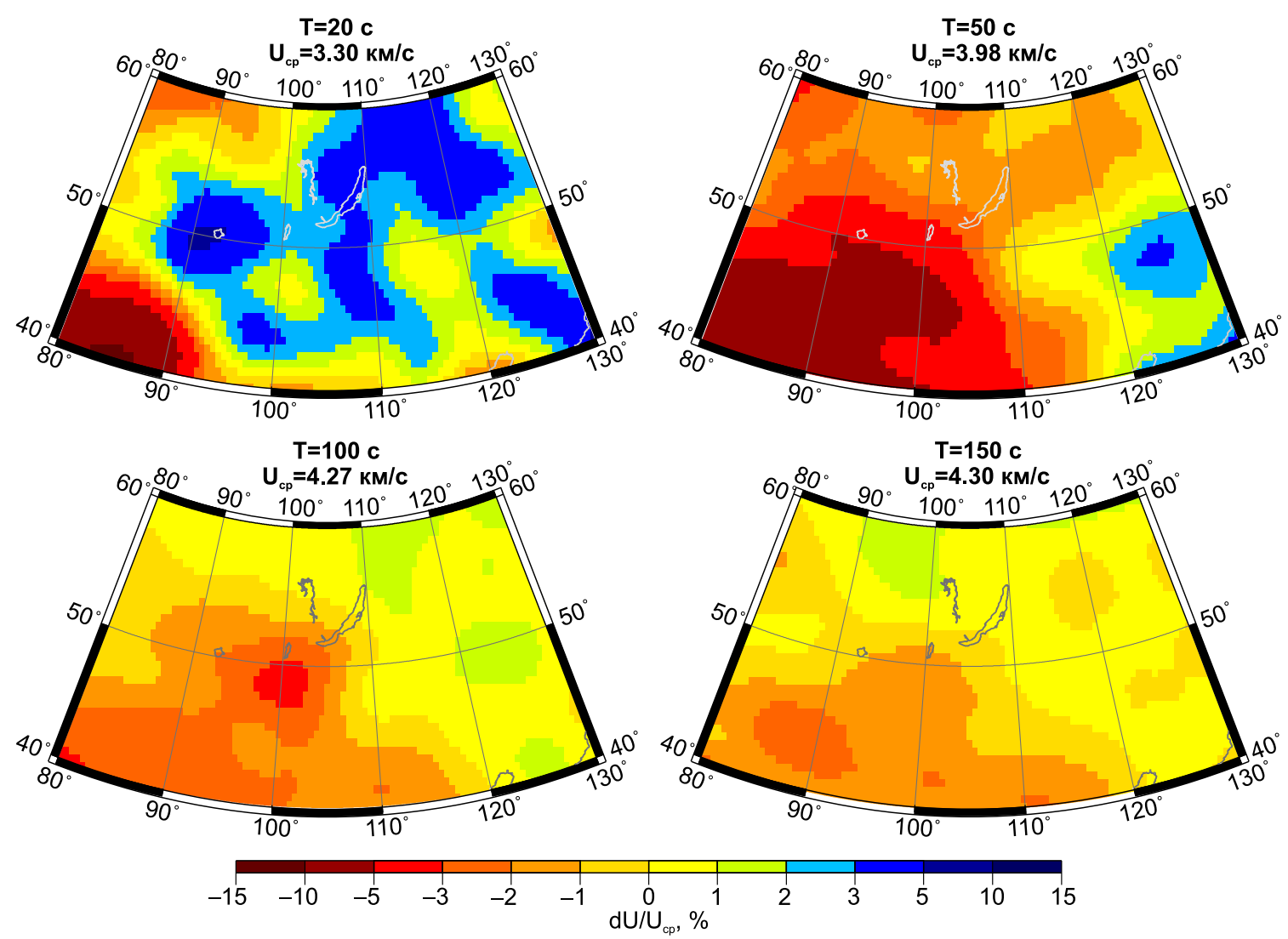

Рис. 5. Карты распределений групповых скоростей волн Лява. Над каждой картой приведен соответствующий период и средняя групповая скорость $\left(\mathrm{U}_{\mathrm{cp}}\right)$.

Fig. 5. Group velocity distribution maps for Love waves. Corresponding periods and average group velocity values ( $\mathrm{U}_{\mathrm{cp}}$ ) are shown above each map.

способностью результатов картирования, различной чувствительностью групповых скоростей волн Рэлея и Лява к параметрам модели среды [Yanovskaya, 2015] и вертикальной анизотропией, про- являющейся в различии скоростей волн $S V$ и $S H$ (рис. 7). Поскольку количество используемых для анализа сейсмических трасс и их конфигурация как для волн Рэлея, так и для волн Лява практически
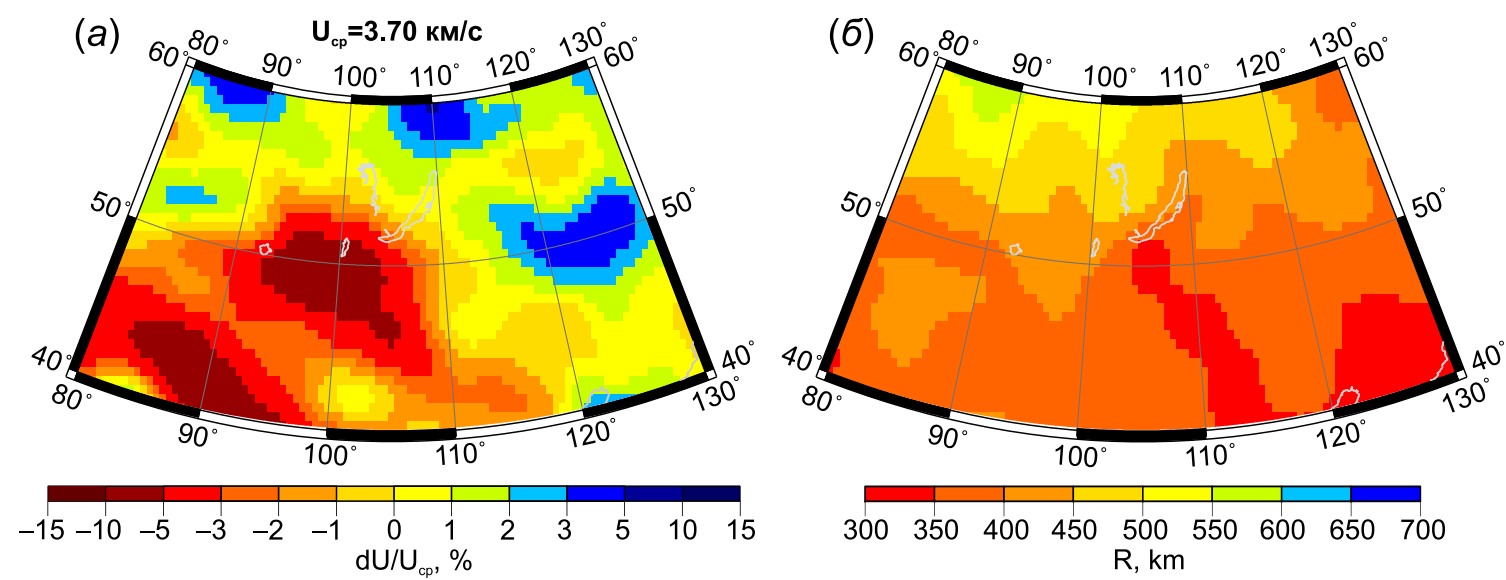

Рис. 6. Пример распределения групповых скоростей волн Рэлея (a) с разрешением (б) для периода 50 с [Kozhevnikov et al., 2014].

Fig. 6. An example of the group velocity distribution for Rayleigh waves (a), and the resolution (6) for the period from $50 \mathrm{~s}$ [Kozhevnikov et al., 2014]. 

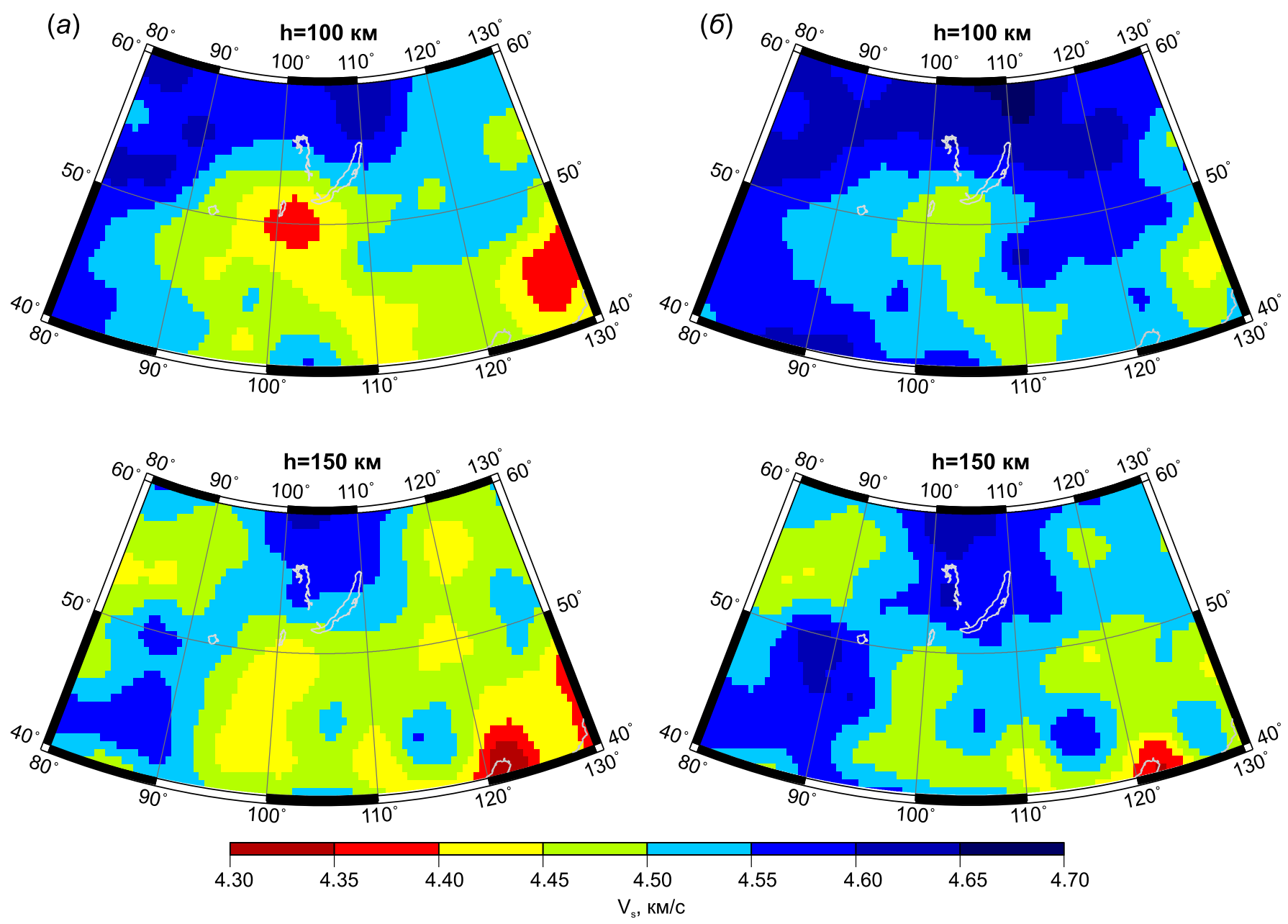

Рис. 7. Примеры распределений скоростей волн $S V(a)$ и $S H(б)$ на глубинах 100 и 150 км.

Fig. 7. Examples of the distribution of $S V$ - and $S H$-wave velocities ( $a$ and 6 , respectively) at the depths of 100 and $150 \mathrm{~km}$.

одинаковы, горизонтальное разрешение на соответствующих периодах также различается незначительно (см. рис. 3; рис. 6, б). Примеры распределений скоростей волн $S V$ и $S H$ на различных глубинах (рис. 7), полученные в результате инверсии результатов картирования групповых скоростей волн Рэлея и Лява, указывают на существенные различия в скоростях распространения этих волн, причем для большей части рассматриваемой территории $\mathrm{V}_{\mathrm{SH}}>\mathrm{V}_{\mathrm{SV}}$, а различия в конфигурации и интенсивности выявленных аномалий скоростей свидетельствуют о неоднородности анизотропных свойств вещества мантии в различных в тектоническом отношении регионах, что более наглядно продемонстрировано на картах распределений коэффициента вертикальной анизотропии (рис. 8).

Кроме того, приведенные распределения скоростей поперечных волн (см. рис. 7) совместно с двумерными скоростными разрезами из работы [Seredkina et al., 2016] позволяют дать оценки мощности литосферы и астеносферы под различными регионами исследуемой области. Так, под высокогорными сооружениями Южной Сибири и Западной Монголии кровля астеносферы находится в непосредственной близости к подошве коры. При переходе к платформенным и субплатформенным структурам она плавно погружается, достигая глубин 200 км под Сибирской платформой. Мощность астеносферы под структурами МонголоОхотского складчатого пояса варьируется в пределах 80-150 км, тогда как под Сибирской платформой она утоняется до 50 км.

Из рис. 4 и рис. 8 видно, что анизотропия наблюдается до глубины около 250 км, что приблизительно соответствует нижней границе астеносферы [Seredkina et al., 2016]. Максимальные значения коэффициента анизотропии приурочены к интервалу глубин от подошвы коры до 150 км, т. е. к мантийной части литосферы и к астеносфере, что несколько выше полученных ранее с бо́льшим осреднением оценок интервала глубин 100-200 км [Yanovskaya, Kozhevnikov, 2006]. Земная кора (см. 

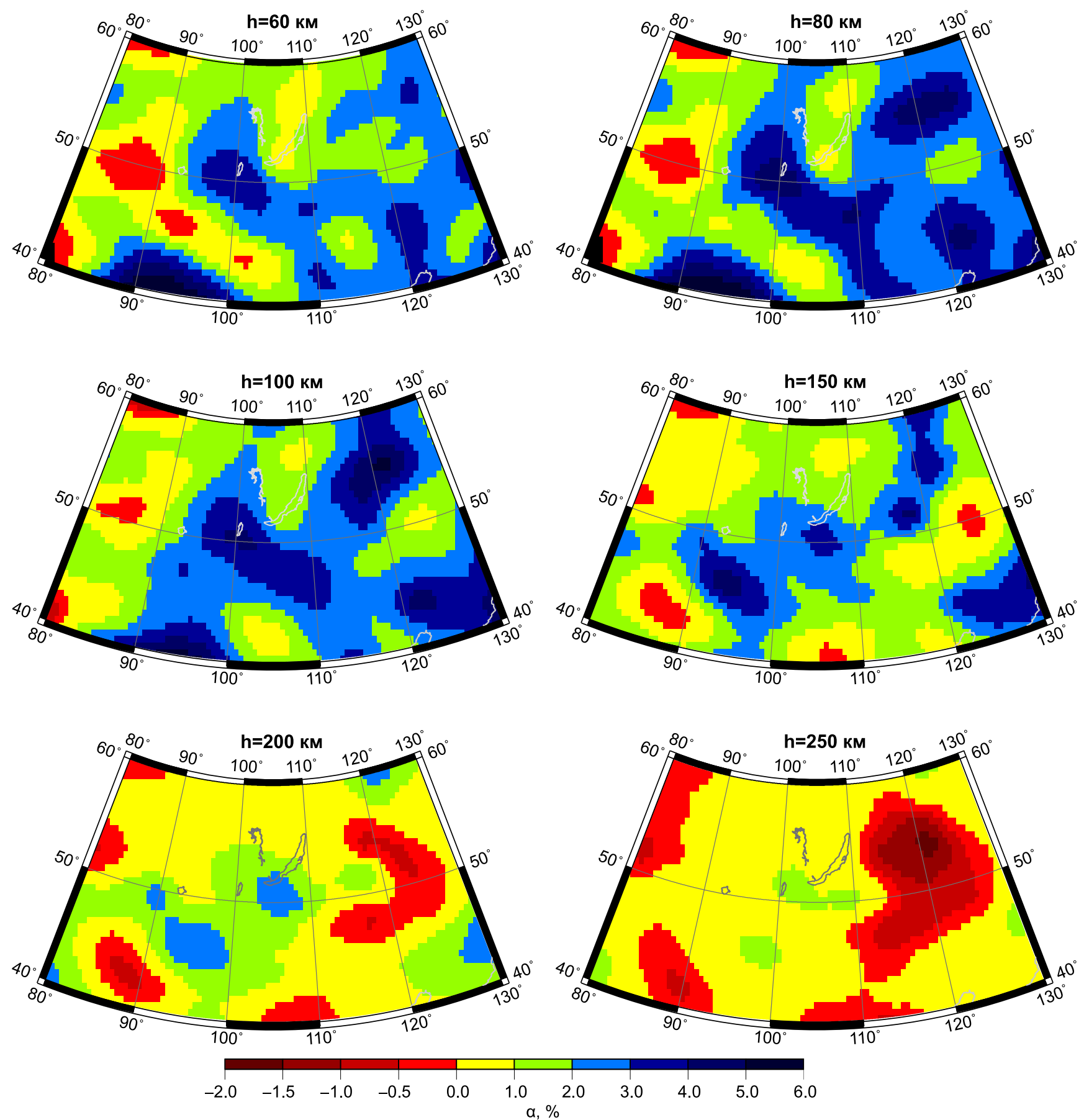

Рис. 8. Карты распределений коэффициента вертикальной анизотропии. Над каждой картой приведена соответствующая глубина.

I Fig. 8. Distribution maps of the vertical anisotropy coefficient. Corresponding depth values are shown above each map.

рис. 4) также обладает ненулевой вертикальной анизотропией, вероятно, обусловленной различными процессами в ее верхней и нижней части [Meissner et al., 2002]. Однако подробное рассмотрение этого вопроса выходит за рамки данного исследования.

Полученные результаты свидетельствуют о наличии анизотропных свойств в мантии до глубины 250 км под южной частью Сибирской платформы $(\alpha=1 \ldots 2 \%)$, что согласуется с данными исследований стабильных областей Земли различными методами [Fouch, Rondenay, 2006]. Для тектонически активных структур Монголо-Охотского складчатого пояса характерно более сложное распределение анизотропных свойств как в горизонтальном, так и в вертикальном направлении. Например, интересной особенностью является локальный максимум (до 6 \%) коэффициента анизотропии на границе се- 
веро-восточного фланга Байкальского рифта и Станового хребта, проявляющийся от подошвы коры до глубины 150 км.

Сопоставляя карты распределений коэффициента анизотропии (рис. 8) и распределений скоростей $S$-волн на различных глубинах (см. рис. 7) [Seredkina et al., 2016], можно заметить, что тектонически активные регионы характеризуются высокими значениями коэффициента анизотропии и пониженными значениями скоростей $S$-волн. Анизотропия в этих низкоскоростных областях, как предполагается, вызвана влиянием термоупругих деформаций на свойства вещества мантии [Yanovskaya, Kozhevnikov, 2006; Chen et al., 2009].

Существование отрицательной вертикальной анизотропии в мантии долгое время подвергалось сомнению [Yanovskaya, Kozhevnikov, 2006; Dziewonski, Anderson, 1981; Shapiro, Ritzwoller, 2002], однако было показано в более поздних исследованиях разного масштабного уровня. Так, согласно [Zhou et al., 2006], для всей Земли в целом на глубинах более 220 км происходит смена знака коэффициента вертикальной анизотропии, возможно, обусловленная изменением характера течения вещества с горизонтального в астеносфере на вертикальное в более глубоких слоях мантии. Области с наиболее сильной отрицательной анизотропией (до $-5 \%$ ) приурочены к океанам и бассейнам окраинных морей, в то время как на континентах коэффициент вертикальной анизотропии принимает значения до $-1 \ldots-2 \%$, что согласуется с результатами данного исследования (рис. 8) и с работой [Yanovskaya, Akchurin, 2009]. В тектонически активных областях
Земли отрицательная анизотропия может наблюдаться не только на глубинах более 220 км, но и в подкоровых слоях мантии, что было показано в работе [Chen et al., 2009] на примере Тибета и сопредельных регионов. В нашем случае аналогичная ситуация реализуется в высокогорных районах Монгольского и Гобийского Алтая.

\section{4. ЗАКЛЮЧЕНИЕ}

В результате проведенного исследования было показано, что верхняя мантия Центральной Азии характеризуется анизотропными свойствами до глубины около 250 км, причем распределение анизотропных свойств является неоднородным и согласуется с геологическим строением исследуемой территории. Так, в тектонически активных регионах наблюдаются высокие значения коэффициента анизотропии и пониженные значения скоростей $S$-волн. Области с отрицательной вертикальной анизотропией $\left(\mathrm{V}_{\mathrm{SH}}<\mathrm{V}_{\mathrm{SV}}\right)$ наиболее сильно проявлены на глубинах более 200 км. Полученные результаты в дальнейшем могут способствовать построению более детальных и обоснованных геодинамических моделей рассматриваемой территории.

\section{5. БЛАГОДАРНОСТИ}

Работа выполнена при поддержке РНФ, грант № 17-77-10037.

\section{6. ЛИТЕРАTУРА / REFERENCES}

Amante C., Eakins B.W., 2009. ETOP01. 1 Arc-Minute Global Relief Model: Procedures, Data Sources and Analysis. NOAA Technical Memorandum NESDIS NGDC-24. National Geophysical Data Center, NOAA. https://doi.org/ 10.7289/V5C8276M.

Bijwaard H., Spakman W., Engdahl E.R., 1998. Closing the gap between regional and global travel time tomography. Journal of Geophysical Research: Solid Earth 103 (B12), 30055-30078. https://doi.org/10.1029/98JB02467.

Chen Y., Badal J., Zhang Z., 2009. Radial anisotropy in the crust and upper mantle beneath the Qinghai-Tibet Plateau and surrounding regions. Journal of Asian Earth Sciences 36 (4-5), 289-302. https://doi.org/10.1016/j.jseaes. 2009.06.011.

Dziewonski A.M., Anderson D.L., 1981. Preliminary Reference Earth Model. Physics of the Earth and Planetary Interiors 25 (4), 297-356. https://doi.org/10.1016/0031-9201(81)90046-7.

Forsyth D.W., 1975. The early structural evolution and anisotropy of the oceanic upper mantle. Geophysical Journal of the Royal Astronomical Society 43 (1), 103-162. https://doi.org/10.1111/j.1365-246X.1975.tb00630.x.

Fouch M.J., Rondenay S., 2006. Seismic anisotropy beneath stable continental interiors. Physics of the Earth and Planetary Interiors 158 (2-4), 292-320. https://doi.org/10.1016/j.pepi.2006.03.024.

Koulakov I., Bushenkova N., 2010. Upper mantle structure beneath the Siberian craton and surrounding areas based on regional tomographic inversion of P and PP travel times. Tectonophysics 486 (1-4), 81-100. https://doi.org/ 10.1016/j.tecto.2010.02.011.

Kozhevnikov V.M., Seredkina A.I., Solovei O.A., 2014. 3D mantle structure of Central Asia from Rayleigh wave group velocity dispersion. Russian Geology and Geophysics 55 (10), 1239-1247. https://doi.org/10.1016/j.rgg.2014.09.010.

Levshin A.L., Yanovskaya T.B., Lander A.V., Bukchin B.G., Barmin M.P., Ratnikova L.I., Its E.N., 1986. Surface Seismic Waves in a Laterally Inhomogeneous Earth. Nauka, Moscow, 278 p. (in Russian) [Левшин А.Л., Яновская Т.Б., 
Ландер А.В., Букчин Б.Г., Бармин М.П., Ратникова Л.И., Итс Е.Н. Сейсмические поверхностные волны в горизонтально-неоднородной Земле. М.: Наука, 1986. 278 с.].

Li Y., Wu Q., Pan J., Zhang F., Yu D., 2013. An upper mantle S-wave velocity model for East Asia from Rayleigh wave tomography. Earth and Planetary Science Letters 377-378, 367-377. https://doi.org/10.1016/j.epsl.2013.06.033.

Meissner R., Mooney W.D., Artemieva I., 2002. Seismic anisotropy and mantle creep in young orogens. Geophysical Journal International 149 (1), 1-14. https://doi.org/10.1046/j.1365-246X.2002.01628.x.

Pandey S., Yuan X., Debayle E., Priestley K., Kind R., Tilmann F., Li X., 2014. A 3D shear-wave velocity model of the upper mantle beneath China and the surrounding areas. Tectonophysics 633, 193-210. https://doi.org/10.1016/j.tecto. 2014.07.011.

Ritzwoller M.H., Levshin A.L., 1998. Eurasian surface wave tomography: group velocities. Journal of Geophysical Research: Solid Earth 103 (B3), 4839-4878. https://doi.org/10.1029/97JB02622.

Seredkina A.I., Kozhevnikov V.M., Melnikova V.I., Solovey O.A., 2016. Seismicity and S-wave velocity structure of the crust and the upper mantle in the Baikal rift and adjacent regions. Physics of the Earth and Planetary Interiors 261 (Part B), 152-160. https://doi.org/10.1016/j.pepi.2016.10.011.

Shapiro N.M., Ritzwoller M.H., 2002. Monte-Carlo inversion for a global shear velocity model for the crust and upper mantle. Geophysical Journal International 151 (1), 88-105. https://doi.org/10.1046/j.1365-246X.2002.01742.x.

Trampert J., Woodhouse J., 2003. Global anisotropic phase velocity maps for fundamental mode surface waves between 40 and 150 s. Geophysical Journal International 154 (1), 154-165. https://doi.org/10.1046/j.1365-246X.2003. 01952.x.

Villaseñor A., Ritzwoller M.H., Levshin A.L., Barmin M.P., Engdahl E.R., Spakman W., Trampet J., 2001. Shear velocity structure of Central Eurasia from inversion of surface wave velocities. Physics of the Earth and Planetary Interiors 123 (2-4), 169-184. https://doi.org/10.1016/S0031-9201(00)00208-9.

Yanovskaya T.B., 2001. A surface wave tomography method based on the Backus-Gilbert approach. In: Problems of lithosphere dynamics and seismicity. Computational Seismology, vol. 32. GEOS, Moscow, p. 11-26 (in Russian) [Яновская Т.Б. Развитие способов решения задач поверхностно-волновой томографии на основе метода Бэйкуса-Гильберта // Вычислительная сейсмология. Вып. 32. Проблемы динамики литосферы и сейсмичности. М.: ГЕОС, 2001. С. 11-26].

Yanovskaya T.B., 2015. Surface-Wave Tomography in Seismological Studies. Nauka, St. Petersburg, 167 p. (in Russian) [Яновская Т.Б. Поверхностно-волновая томография в сейсмологических исследованиях. СПб.: Наука, 2015. 167 c.].

Yanovskaya T.B., Akchurin K.R., 2009. Anisotropy of the upper mantle of the Asian continent from the phase and group velocities of the Rayleigh and Love waves. In: V.N. Troyan, N.I. Uspensky, A.K. Sarayev (Eds.), Issues of geophysics, vol. 42. Publishing House of the St. Petersburg University, St. Petersburg, p. 3-11 (in Russian) [Яновская Т.Б., Акчурин К.Р. Анизотропия верхней мантии Азиатского континента по данным фазовых и групповых скоростей волн Рэлея и Лява // Вопросы геофизики. Вып. 42 / Ред. В.Н. Троян, Н.И. Успенский, А.К. Сараев. СПб.: Изд-во Санкт-Петербургского университета, 2009. С. 3-11].

Yanovskaya T.B., Antonova L.M., Kozhevnikov V.M., 2000. Lateral variations of the upper mantle structure in Eurasia from group velocities of surface waves. Physics of the Earth and Planetary Interiors 122 (1-2), 19-32. https:// doi.org/10.1016/S0031-9201(00)00184-9.

Yanovskaya T.B., Kozhevnikov V.M., 2003. 3D S-wave velocity pattern in the upper mantle beneath the continent of Asia from Rayleigh wave data. Physics of the Earth and Planetary Interiors 138 (3-4), 263-278. https://doi.org/ 10.1016/S0031-9201(03)00154-7.

Yanovskaya T.B., Kozhevnikov V.M., 2006. Anisotropy of the upper mantle of the Asian continent according to the group velocities of Rayleigh and Love waves. Geologiya i Geofizika (Russian Geology and Geophysics) 47 (5), 622-629.

Zhao D., Lei J., Inoue T., Yamada A., Gao S.S., 2006. Deep structure and origin of the Baikal rift zone. Earth and Planetary Science Letters 243 (3-4), 681-691. https://doi.org/10.1016/j.epsl.2006.01.033.

Zhou Y., Nolet G., Dahlen F.A., Laske G., 2006. Global upper-mantle structure from finite-frequency surface-wave tomography. Journal of Geophysical Research: Solid Earth 111 (B4), B04304. https://doi.org/10.1029/2005JB003677.

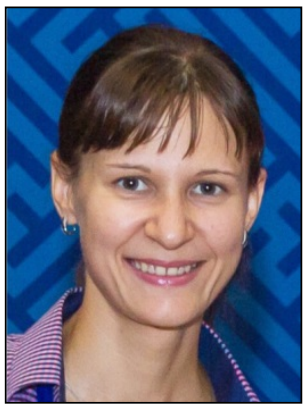

Алена Игоревна Середкина, канд. физ.-мат. наук, с.н.с.

Институт земной коры СО РАН

664033, Иркутск, ул. Лермонтова, 128, Россия

e-mail: ale@crust.irk.ru

Alena I. Seredkina, Candidate of Physics and Mathematics, Senior Researcher

Institute of the Earth's Crust, Siberian Branch of RAS

128 Lermontov street, Irkutsk 664033, Russia

e-mail: ale@crust.irk.ru 


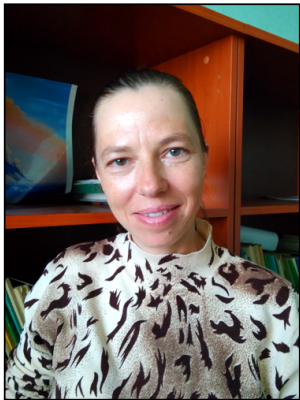

Оксана Анатольевна Соловей, канд. геол.-мин. наук, н.с.

Институт земной коры СО РАН

664033, Иркутск, ул. Лермонтова, 128, Россия

e-mail: Solovey@crust.irk.ru

Oksana A. Solovey, Candidate of Geology and Mineralogy, Researcher Institute of the Earth's Crust, Siberian Branch of RAS

128 Lermontov street, Irkutsk 664033, Russia

e-mail: Solovey@crust.irk.ru 